\title{
DUPLICATION OF THE ILEUM
}

\author{
BY \\ W. S. KILLPACK \\ From the Pathology Department, King Edward Memorial Hospital, London
}

(RECEIVED FOR PUBLCATION JUNe 12, 1952)

Examples of cystic developmental abnormalities of the intestinal canal have been reported with increasing frequency during the past few years (Barrett, 1951; Aitken, 1950; Wooler, 1950; Webb, 1950).

Frequently these structures, which lie in intimate contact with the digestive tube, are the cause of an abdominal emergency with a characteristic clinical picture differing from that of an obstruction of the more normal type (Ladd and Gross, 1941). It is for this reason that anatomical descriptions are few and incomplete, as the associated strangulation with superadded infection obliterates the detail. The incidental discovery, therefore, during the necropsy of a newborn premature infant of an intestinal duplication has permitted the study of one of these developmental abnormalities in the intact state.

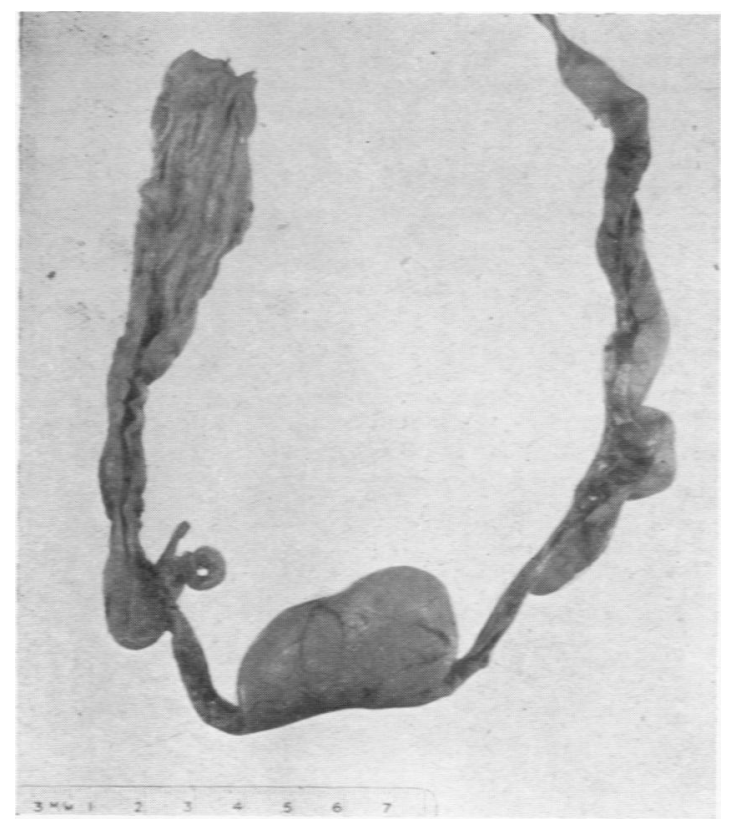

Fig. 1.-Anterior view of cyst showing relation to ileo-caecal valve $\times 0.5$.

\section{Necropsy Findings}

A male infant weighing $4 \frac{1}{2} \mathrm{lb}$. was born six weeks prematurely and died two days later of atelectasis. An elongated cystic swelling was found on the mesenteric border of the ileum near the ileo-caecal valve. Unfortunately the mesentery was not kept intact, the bowel being removed from the body by cutting through the mesentery fairly close to the viscus, and therefore the actual arterial supply could not be investigated.

The cyst, which measured $6 \cdot 3 \cdot 2 \times 2 \cdot 5 \mathrm{~cm}$. before fixation, was tense, and lay between the two layers of the mesentery, with its distal extremity $5 \mathrm{~cm}$. from the ileo-caecal valve (Fig. 1). The terminal mesenteric vessels could be seen coursing over its surface in a radial fashion (Fig. 2). The cyst contents were

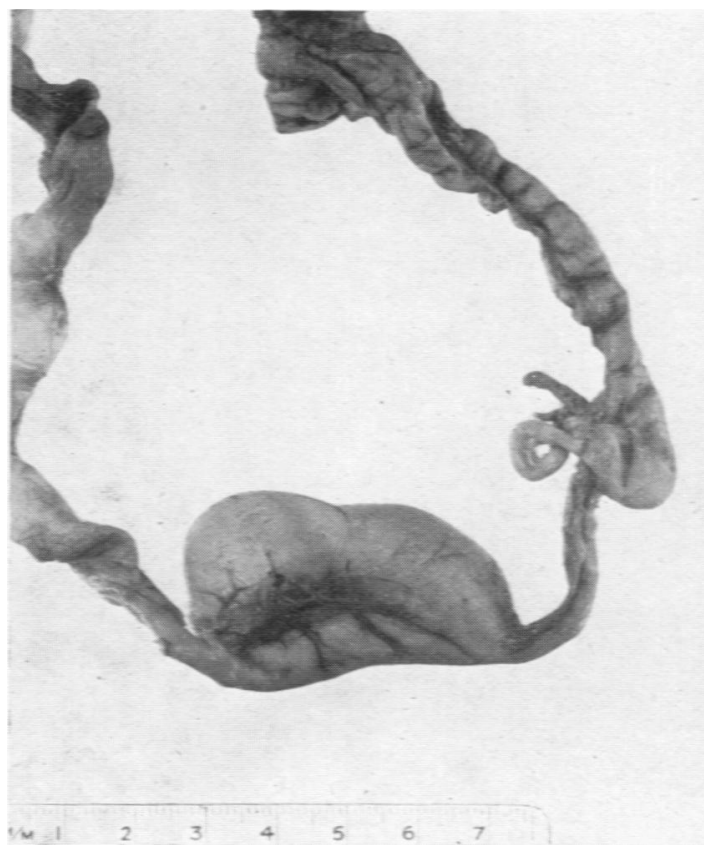

Fug. 2.-Postero-inferior view of cyst showing terminal mesenteric vessels coursing over surface $\times 0.75$. 


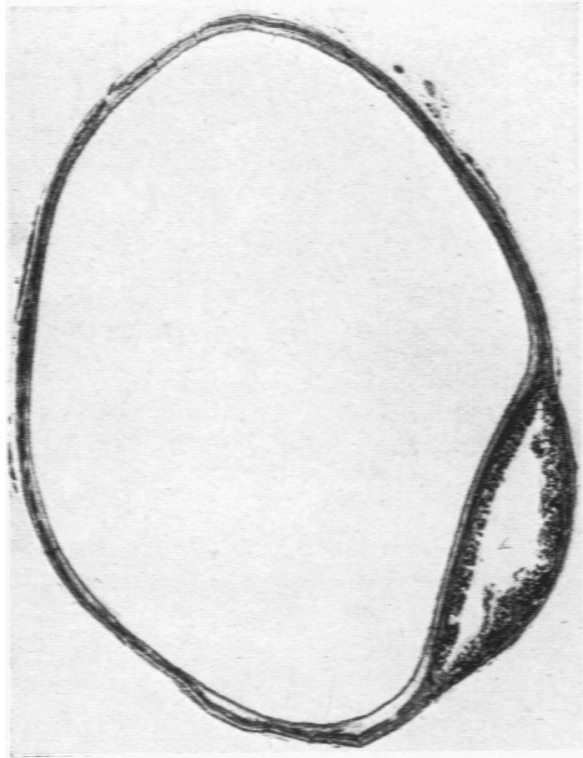

FIG. 3.-Photomicrograph of cross section of cyst and ileum. Haematoxylin and eosin $\times 3 \cdot 5$.

aspirated with difficulty through a wide-bore needle and replaced with $10 \%$ formol saline. The fluid was found to be glairy, clear and colourless, and contained a quantity of mucin. As the adjacent bowel contained meconium, the cyst could not be regarded as a diverticulum.

Some of the fluid was mixed with saline and centrifuged. The deposit consisted of a small number of ciliated columnar epithelial cells, round cells and amorphous debris. After thorough fixation the specimen was frozen and a slice, $0 \cdot 5$ $\mathrm{cm}$. thick, cut for histological examination from the middle of both cyst and adjacent ileum. It was noted that the cyst lining was quite smooth to the naked eye and also that the lumen of the ileum, though apparently normal in size, was distorted in outline by the distended cyst to which it was intimately connected (Fig. 3).

Histological Examination. It was immediately apparent that cyst and bowel were separate structures though sharing a common longitudinal muscle coat (Fig. 4). Mucous membrane, muscularis mucosae, submucosa and circular muscle coats of the bowel were normal (Fig. 5). The cyst had a distinct mucosa composed of stratified polygonal, almost squamous, cells, the most superficial of which formed a continuous layer of ciliated epithelium (Fig. 6). There was a prominent muscularis mucosae, two or three times the thickness of that of the bowel, and a well developed independent
FiG. 4.-Photomicrograph of part of site of fusion of cyst with bowel. Iron haematoxylin and Van Gieson $\times 9 \cdot 5$ 
have their origin very early in embryonic life, and, particularly in. connexion with tubular structures, are commonplace in surgery and pathology. The gross features in their development will not, therefore, be discussed here. However, a striking feature of the duplication cyst in this case was the continuous lining of ciliated epithelium. Normally the only part of the human digestive tube in which this type of epithelium is found is in the oesophagus and then only for a brief period in foetal life. Ciliated cells have been found at the $44 \mathrm{~mm}$. stage, and they form a continuous layer by the eleventh week (65 mm. stage) to disappear slowly by desquamation towards the end of the sixth month $(210 \mathrm{~mm}$. stage). The histogenesis of the epithelial lining of the oesophagus has been summarized recently by Raeburn (1951) who describes the persistence of islands of ciliated epithelium in a woman of 35 years. Epithelium of the type lining this cyst is illustrated by Patten (1946) and appears to be normal for the 13-week-old foetus (85 mm. stage).

There appears to be no reference in the literature to the certain occurrence of ciliated epithelium elsewhere in the normal digestive tube, though the early embryologists believed that the embryonic gastric mucosa was ciliated, on the basis of finding ciliated cells in the gastric contents. It seems certain, however, that these had been carried down from the oesophagus. Keibel and Mall (1910-12) found no evidence of gastric ciliation in their collection of embryos.

The heteropia described here is no less anomalous than the mucosa of gastric type commonly found in remains of the vitello-intestinal canal, or in the jejunal duplication described by Wooler (1950).

\section{Discussion}

The criteria laid down by Ladd and Gross (1941) for alimentary duplications may be summarized here as they are well exemplified in the present case: contiguity with, and firm adherence to, part of the digestive tube; a smooth muscle coat, usually of two layers; mucosal lining or epithelium similar to that of some part of the alimentary tract. 


\section{Addendum}

Since this paper was written Potter (1952) has described a further example of ileum duplex. The arrangement of the musculature is illustrated and is similar to that demonstrated here, except that the mucosal lining of the cystic portion is of small gut type. Potter encountered alimentary tract duplications only twice in over 9,000 foetal and neonatal necropsies.

\section{REFERENCES}

Aitken, J. (1950). Brit. J. Surg., 37, 349.

Barrett, J. C. (1951). Brit. med. J., 2, 889.

Keibel, F. and Mall, F. P. (1910-12). Manual of Human Embryology. J. B. Lippincott \& Co., Philadelphia.

Ladd, W. E and Gross, R. E. (1941). Abdominal Surgery of Infancy and Childhood, 1st ed. W. B. Saundess, Philadelphia

Patten, B. M. (1946). Human Embryology, p. 472 . The Blakiston Co., Philadelphia

Potter, E L (1952). Pathology of the Fetus and the Newborn. The Yearbook Publi-hets Inc., Chicago.

Raeburn, C. (1951). J. Path. Bact., 63, 157.

Webb, J. R. D. (1950). Proc. roy. Soc. Med., 43, 613.

Wooler, G. H. (1950). Brit. J. Surg., 37, 356. 